\title{
On the forward solution of electroencephalography and magnetoencephalography
}

\author{
Pia Kemppainen and Maria Peters \\ Biomagnetic Centre, Low Temperature Division, University of Twente, PO Box 217, \\ 7500 AE Enschede, The Netherlands
}

\begin{abstract}
The collocation method combined with a serial expansion of the electric potential is presented as an alternative way of solving the boundary integral equations of electroencephalography and magnetoencephalography.
\end{abstract}

\section{Introduction}

In order to use electroencephalography (EEG) and/or magnetoencephalography (MEG) for the localisation of active (evoked/spontaneous) areas, a mathematical model is needed to describe how the neuronal activity generates a current distribution. This distribution results in an electrical potential and a magnetic field. In the model, the shape and the conductivity distribution of the head need to be prescribed. Usually it is assumed that a small activated area can be described by a current dipole embedded in a conducting medium.

The electrical potential and the magnetic field can be calculated by solving the Maxwell equations within the model. This is called the forward problem. The localisation of the sources is called the inverse problem: everything except the source parameters is known. By comparing the measured values of the electrical potential/magnetic field with the values of the forward problem solution, the source is iteratively moved into such a position that the measured data fit the forward solution data optimally.

In this article a method is described for solving the forward problem (Barnard et al 1967) for a model in which the head consists of homogeneous, isotropic compartments. Usually, the standard boundary element method (BEM) is used, but if the difference between the conductivities of the cerebrospinal fluid and the skull is assumed to be high, the BEM gives rise to numerical problems, making this method inappropriate for the inverse solution. We present an alternative method: the shapes of the interfaces separating different tissues are given in an analytical two-parameter form. The electrical potential (which is also needed for the evaluation of the magnetic field) is expanded in a finite series of independent base functions. By increasing the number of parameters in both the surface and potential descriptions, the accuracy of the forward solution can be improved. The influence of the number of parameters was tested in a spherical model because, in this simplified case, analytical results exist and can be compared with the numerical ones.

\section{The basic formulae}

In order to compute the electromagnetic fields generated by neural currents, it is sufficient to use the Maxwell equations in their quasistatic form,

$$
\begin{array}{lrl}
\nabla \cdot \boldsymbol{E}=\varrho / \epsilon_{0} & \nabla \times \boldsymbol{E}=0 \\
\nabla \cdot \boldsymbol{B}=0 & \nabla \times \boldsymbol{B}=\mu_{0} \boldsymbol{J}
\end{array}
$$


where $\boldsymbol{E}$ denotes the electric field, $\boldsymbol{B}$ the magnetic field and $\varrho$ a charge distribution. The current density $\boldsymbol{J}$ is divided into two terms, $\boldsymbol{J}=\boldsymbol{J}^{\mathrm{p}}+\sigma \boldsymbol{E}$, where the primary current $\boldsymbol{J}^{\mathrm{p}}$ models the cause of the volume currents $\sigma \boldsymbol{E}$.

Charge does not accumulate in the tissue, so $\nabla \cdot \boldsymbol{J}=0$. By writing $\boldsymbol{E}=-\nabla V$, we obtain a partial differential equation for the potential $\mathrm{V}$

$$
\nabla \cdot \boldsymbol{J}=0 \Leftrightarrow \nabla \cdot(\sigma \nabla \mathrm{V})=\nabla \cdot J^{\mathrm{p}}
$$

Once the potential is known, the magnetic field can be calculated from the Biot-Savart law

$$
\boldsymbol{B}=\left(\mu_{0} / 4 \pi\right) \int J(r) \times \nabla^{\prime} R^{-1} \mathrm{~d} v^{\prime}
$$

where the interval of integration extends over the entire space. This solution also satisfies equations (1).

\section{Boundary element formulation}

The conductor model consists of layers representing the different tissues, e.g. brain, skull and scalp. The boundaries of the compartments are closed and non-intersecting. The conductivity $\sigma$ is assumed to be piecewise constant.

The surfaces will not be discretised, but instead the coordinates on each will be presented as continuous two-parametric functions

$$
\mathbf{r}=(x(u, v), y(u, v), z(u, v))
$$

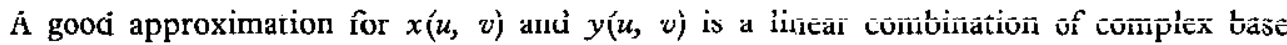
functions

$$
\begin{aligned}
f_{z}(u, v) & =\sum \beta \Psi(u, v) \\
x & =\operatorname{Re} f_{z}(u, v) \\
y & =\operatorname{Im} f_{z}(u, v) \\
z & =z(u, v)
\end{aligned}
$$

For example, all points on a sphere satisfy the relations (5), when $f$ and $z$ are

$$
\begin{aligned}
f_{z} & =\sum_{n} \sum_{m} \beta_{n m} \sin (n \theta) \exp (\mathrm{i} m \phi) \\
Z & =R \cos \theta
\end{aligned}
$$

and only the first coefficient is non-zero: $\beta_{11}=R$.

Surfaces remain continuous and differentiablc in the model. The unknown coefficients in equation (5) will be found by a least-squares fit; a set of sample points is obtained, for example, from MRI images.

The potential distribution is continuous everywhere, but its gradient, the electric field, is not. The normal component of the current density, however, must be continuous across the boundaries:

$$
\sigma_{j}^{-} \mathbf{n}_{-} \cdot \nabla V^{-}=\sigma_{j}^{+} n_{+} \cdot \nabla V^{+}
$$

Equations (7) form the boundary conditions for equation (2).

With these assumptions and conditions it is possible to formulate (2) as an integral equation of the potential $V$ (Barnard et al 1967):

$$
\left(\sigma_{j}^{-}+\sigma_{j}^{+}\right) V(\boldsymbol{r})=2 V_{0}(\boldsymbol{r})+\frac{1}{2} \pi \sum_{k}\left(\sigma_{k}^{-}-\sigma_{k}^{+}\right) \int_{S_{k}} V\left(\boldsymbol{r}^{\prime}\right) \frac{\boldsymbol{r}^{\prime}-\boldsymbol{r}}{\left|\boldsymbol{r}^{\prime}-\boldsymbol{r}\right|^{3}} \cdot \mathrm{d} \boldsymbol{S}
$$


When the potential on each surface is expressed as a series (Gonzalez et al 1989)

$$
V(\boldsymbol{r})=\sum_{m} c_{m}^{j} \Psi_{m}(\boldsymbol{r}) \quad \boldsymbol{r} \in \mathbf{S}_{j}
$$

and this representation is inserted into (9), the problem changes into

$$
\left(\sigma_{j}^{-}+\sigma_{j}^{+}\right) \sum_{m} c_{m}^{j} \Psi_{m}(\boldsymbol{r})=2 V_{0}(\boldsymbol{r})+\frac{1}{2} \pi \sum_{k}\left(\sigma_{k}^{-}-\sigma_{k}^{+}\right) \sum_{k} c_{m}^{k} I_{j k}\left(\Psi_{m}\right)
$$

where

$$
\mathrm{I}_{\mathrm{jk}}\left(\Psi_{m}\right)=\int_{S_{k}} \Psi_{m}\left(\boldsymbol{r}^{\prime}\right) \frac{\boldsymbol{r}^{\prime}-\boldsymbol{r}}{\left|\boldsymbol{r}^{\prime}-\boldsymbol{r}\right|^{3}} \cdot \mathrm{d} \boldsymbol{S}^{\prime} \quad \boldsymbol{r} \in \mathbf{S}_{j}
$$

In order to determine the unknowns $c_{m}^{j}$, equation (11) is evaluated on each surface on a set of so-called collocation points. In the case of a two-layer model, the linearised problem reduces to

$$
\begin{aligned}
& {\left[\begin{array}{rr}
\Psi-\gamma_{11} I_{11}(\Psi) & -\gamma_{12} I_{12}(\Psi) \\
-\gamma_{21} I_{21}(\Psi) & \Psi-\gamma_{22} I_{22}(\Psi)
\end{array}\right]\left[\begin{array}{l}
c^{1} \\
c^{2}
\end{array}\right]=\left[\begin{array}{c}
\eta_{1} V_{0}^{1} \\
\eta_{2} V_{0}^{2}
\end{array}\right]} \\
& \mathbf{A c}=\mathbf{s}
\end{aligned}
$$

where the matrix $\mathbf{A}$ contains all geometrical information of the problem, while $\mathbf{s}$ defines the source model. The unknown vector $c$ is solved in a least squares sense

$$
\mathbf{c}=\mathbf{A}^{+} \mathbf{s}
$$

$\mathbf{A}^{+}$denotes the pseudo-inverse of $\mathbf{A}$. Once the coefficients have been fixed, the potential can be extrapolated at any point on a surface.

For the evaluation of the magnetic field $\boldsymbol{B}$ at a point $\boldsymbol{r}$, additional integrals of the potential distribution will be needed:

$$
\boldsymbol{B}(\boldsymbol{r})=\boldsymbol{B}_{0}(\boldsymbol{r})+\mu_{0} / 4 \pi \sum_{k}\left(\sigma_{k}^{-}+\sigma_{k}^{+}\right) \sum_{m} c_{m}^{k} \boldsymbol{Q}_{k}\left(\Psi_{m}\right)
$$

where

$$
\boldsymbol{Q}_{k}\left(\Psi_{m}\right)=\int_{S_{k}} \Psi_{m}\left(\boldsymbol{r}^{\prime}\right) \frac{\boldsymbol{r}-\boldsymbol{r}^{\prime}}{\left|\boldsymbol{r}-\boldsymbol{r}^{\prime}\right|^{3}} \cdot \mathrm{d} \boldsymbol{S}^{\prime}
$$

The field component, which a magnetometer measures, is then

$$
\boldsymbol{B}(\boldsymbol{r}) \cdot \mathbf{e}=\boldsymbol{B}_{0}(\boldsymbol{r}) \cdot \mathbf{e}+\mathbf{c}^{\mathrm{T}} \mathbf{Q}=\boldsymbol{B}_{0}(\boldsymbol{r}) \cdot \mathbf{e}+\mathbf{s}^{\mathrm{T}} \mathbf{A}+\mathrm{T} \mathbf{Q}=B_{0}(\boldsymbol{r}) \cdot e+\mathbf{s}^{\mathrm{T}} \mathbf{D}
$$

where $\mathbf{e}$ is the unit normal vector of the pick-up loop. The matrix $\mathbf{D}$ remains the same as long as the measurement point $\boldsymbol{r}$ does not change. If this formulation is used to solve the inverse problem of EEG-MEG, on each trial only the source vectors $\boldsymbol{B}_{0}$ and $\mathbf{s}$ will have to be re-evaluated. 


\section{Features of the method}

It is of practical interest to estimate how many terms in the expansion (9) will be needed in order to reach a precision $p$. The answer, however, depends on the choice of the minimisation criterion. If the true potential can be expressed as an infinite series

$$
V=\sum_{i=1}^{\infty} a_{i} \Psi_{i}
$$

where the coefficients $a_{i}$ are known analytically and the base $\Psi$ is orthogonal, the finite series

$$
\bar{V}_{N}=\sum_{i=1}^{N} a_{i} \Psi_{i}
$$

( $N$ are the first coefficients from the infinite series) minimises the least squares error

$$
\epsilon_{2}=\int\left(V-V_{N}\right)^{2} \mathrm{~d} S
$$

Thus a numerical approximation $V_{N}^{\prime}$ has an error $\epsilon_{2}^{\prime} \geqslant \bar{\epsilon}_{2}$. On the other hand, if the error is measured as the maximum pointwise deviation

$$
\epsilon_{1}=\max _{\boldsymbol{r}}\left|V(\boldsymbol{r})-V_{N}(\boldsymbol{r})\right|
$$

then $\overline{V_{N}}$ may or may not be the optimal solution, but it is obvious, that its maximum jüintiwise deviatatioñ

$$
\bar{\epsilon}_{1}=\max _{\boldsymbol{r}}\left|V(\boldsymbol{r})-V_{N}(\boldsymbol{r})\right|
$$

serves as an upper bound: $\epsilon_{1} \geqslant \bar{\epsilon}_{1}$.

For a concentric-sphere model, the analytic expression of the potential can be found, for example, in Nunez (1981). Since the potential distribution is a linear combination of the point source potentials, it is enough to construct the error estimate for a current dipole. Due to the rotational symmetry, the dipole can always be positioned on the $z$-axis with its moment in the $x z$ plane.

For a two-layer model the number of base functions and the corresponding accuracy in the potential is presented in table 1 for a radial dipole at three depths. The error was measured as the deviation at the point $(0,0,1)$, when the dipole was at $\left(0,0, r_{\mathrm{Q}}\right)\left(\epsilon_{1}\right.$ error).

Table 1. The approximate precision $p\left(N, r_{Q}\right)$ (in per cent) of the potential for a two-layer spherical model, in which the inner radius was 0.96 , uuter radius 1.00 and relative conductivities 1.0 and 0.05 , respectively. The radial dipole was set to $\left(0,0, r_{Q}\right)$ and the potential was calculated at $(0,0,1)$. N is the number of terms in (9).

\begin{tabular}{lccc}
$\begin{array}{l}p,\left(N, r_{\mathrm{Q}}\right) \\
(\%)\end{array}$ & $N=50$ & $N=70$ & $N=90$ \\
\hline$r_{\mathrm{Q}}=0.85$ & 99 & - & - \\
$r_{\mathrm{Q}}=0.90$ & 95 & 99 & - \\
$r_{\mathrm{Q}}=0.93$ & 85 & 95 & 98 \\
\hline
\end{tabular}

In a sphere model, when the source is close to the brain-skull boundary, at least 70 terms are needed to reach $95 \%$ accuracy in the potential of the radial dipole. If a tangential component is added, $N$ increases easily to 150 on each surface. Even more coefficients will be needed in order to compensate for lack of symmetry in a realistic geometry. 
In practice this means that the matrix $\mathbf{A}$ in equation (13) becomes large and ill conditioned for numerical computations. It would be preferable to keep the problem size small, or at least limited and independent of the dipole position.

\section{Discussion}

The basic reason for the increase of $N$, when the dipole approaches a boundary, is the strongly localised nature of the potential distribution. The linear combination of the base functions is required to approximate the potential both at the high-gradient region near the source and at the low-gradient area elsewhere.

In order to improve the solution (9) and yet keep $N$ relatively small, we reformulate the problem slightly. It may be possible to approximate the potential on each surface $S_{j}$ by

$$
V_{j}=W_{j}+R_{j}=W_{j}+\sum_{m} c_{m}^{j} \Psi_{m}
$$

where the initial solution $W_{j}$ would contain the strong gradients and place the maximum and minimum almost correctly. The remainder consists of spatial low-frequency terms, which improve the numerical solution. The functional form of $W$ is likely to be found from the theory for systems of Fredholm integral equations (Pogorzelski 1966).

\section{Acknowledgment}

This work is supported by the Technology Foundation (STW, The Netherlands).

\section{References}

Barnard A C L, Duck I M and Lynn M S 1967 The application of electromagnetic theory to electrocardiology: I Derivation of the integral equations Biophys. $7.7443-62$

Gonzalez S, Grave de Peralta R, Biscay R, Jimenez J C, Pascual R D, Lemagne I and Valdes P A 1989 Projective methods for the magnetic direct problem Advances in Biomagnetism ed S J Williamson, M Hoke, G Stroink and $M$ Kotani (New York: Plenum Press) pp 615-8

Nunez P L 1981 Electric Fields of the Brain (New York: Oxford University Press)

Pogorzelski W 1966 Integral Equations and Their Applications (Oxford: Pergamon Press) 